\title{
Vortex-Antivortex annihilation dynamics in a square mesoscopic superconducting cylinder
}

\author{
Edson Sardella, ${ }^{1}$ Paulo Noronha Lisboa Filho, ${ }^{1}$ Clécio C. de Souza \\ Silva, ${ }^{2}$ Leonardo Ribeiro Eulálio Cabral, ${ }^{2}$ and Wilson Aires Ortiz ${ }^{3}$ \\ 1 UNESP-Universidade Estadual Paulista, Departamento de Física, \\ Faculdade de Ciências, Caixa Postal 473, 17033-360, Bauru-SP, Brazil \\ 2 Departamento de Física, Universidade Federal de Pernambuco, 50670-901, Recife-PE, Brazil \\ ${ }^{3}$ Departamento de Física, Universidade Federal de São Carlos, 13565-905, São Carlos-SP, Brazil
}

(Dated: June 23, 2021)

\begin{abstract}
The dynamics of the annihilation of a vortex-antivortex pair is investigated. The pair is activated magnetically during the run of a simulated hysteresis loop on a square mesoscopic superconducting cylinder with an antidot inserted at its center. We study the nucleation of vortices and antivortices by first increasing the magnetic field, applied parallel to the axis of the sample, from zero until the first vortex is created. A further increase of the field pulls the vortex in, until it reaches the antidot. As the polarity of the field is reversed, an antivortex enters the scene, travels toward the center of the sample and eventually the pair is annihilated. Depending on the sample size, its temperature, and Ginzburg-Landau parameter, the vortex-antivortex encounter takes place at the antidot or at the superconducting sea around it. The position and velocity of the vortex and antivortex singularities were evaluated as a function of time. The current density, magnetization and order parameter topology were also calculated.
\end{abstract}

Achieving a deep understanding of the nucleation and propagation of vortices in real superconductors is a truly complex task, since these entities interact with almost everything: first, with the surface of the specimen, to surpass it; upon entrance, with other vortices that might have already penetrated, and also with defects, which might attract them and even act as pinning centers. Additional difficulties to emulate the problem arise from the fact that vortices generate heat while propagating, what can be harmful to the robustness of the superconducting properties, if not catastrophic, as is the case of vortex avalanches observed in some superconducting films [1, 2, 3, 4, 5, 6]. It is quite common, however, that the existence of pinning potentials represent a beneficial feature, since vortices can thus be prevented from undergoing dissipative motion. An interesting approach to the problem, which enables one to address most specificities without excessive complexity, is to work in the small universe of mesoscopic samples. In such an ambient, one can accommodate the essential ingredients: relatively important surface-to-volume ratio, only a few vortices on scene, and a number of defects - the so-called antidots - usually arranged in a regular pattern. Furthermore, one can study the interaction of an individual vortex-antivortex (V-AV) pair and, eventually, witness their mutual annihilation.

Recently, there has been many studies about V-AV configurations in mesoscopic superconductors (see for instance References [7, 8, 9, 10, 11]). The authors of these references have found that vortices and antivortices may coexist in equilibrium in configurations which look like a V-AV molecule. A somewhat common approach is to assume an a priori configuration and minimize the free energy in terms of some relevant parameter for which the $\mathrm{V}$-AV molecule is a stable configuration. Here, we will focus in a rather different approach concerning more with the dynamics of a V-AV encounter. The aim of the present work is to elucidate the details involved in the process of creation of pairs, following their time-evolution and ultimate disappearance. We opted to do this making no use of a priori assumptions regarding symmetries, but simply varying the applied magnetic field, allowing for the spontaneous nucleation of a vortex and, cycling the field, of an antivortex, which form a pair of interacting entities, whose subsequent time-evolution is monitored.

In this Letter we present the dynamics of the annihilation of a V-AV pair activated magnetically during a short hysteresis loop. The geometry we consider is a square mesoscopic superconducting cylinder with an antidot placed at its center. Simulations are made in the presence of an applied magnetic field parallel to the axis of the cylinder. We study the nucleation of vortices and antivortices by first increasing the magnetic field from zero until the first vortex is created. The field was then decreased toward negative values. During this process the vortex travels heading the center of the sample, is trapped by the antidot and is then annihilated by an antivortex as the polarity of the applied field is reversed. To monitor the whole process, we evaluate the position and velocity of the vortex and antivortex singularities as a function of time. We also calculate the order parameter topology, the current density, and the magnetization. In order to follow the dynamics of the physical quantities we use the gauge-invariant time-dependent Ginzburg-Landau (TDGL) equations. These equations describe the time-evolution of the complex order parameter $\psi$ and the vector potential $\mathbf{A}$, which is related to the local magnetic field through the expression $\mathbf{h}=\boldsymbol{\nabla} \times \mathbf{A}$. 
In the absence of transport currents, these equations read

$$
\begin{aligned}
\eta \frac{\partial \psi}{\partial t} & =-(-i \boldsymbol{\nabla}-\mathbf{A})^{2} \psi+(1-T) \psi\left(1-|\psi|^{2}\right), \\
\beta \frac{\partial \mathbf{A}}{\partial t} & =\mathbf{J}_{s}-\kappa^{2} \boldsymbol{\nabla} \times \mathbf{h},
\end{aligned}
$$

where the supercurrent density is

$$
\mathbf{J}_{s}=(1-T) \Re[\psi(-i \boldsymbol{\nabla}-\mathbf{A}) \psi] .
$$

Here, the distances are measured in units of the coherence length at zero temperature $\xi(0)$; the magnetic field in units of the upper critical field at zero temperature $H_{c 2}(0)$; the temperature in units of the critical temperature $T_{c}$; the time is in units of the characteristic time $t_{0}=\pi \hbar / 8 K_{B} T_{c}$ [12]; $\kappa$ is the Ginzburg-Landau parameter; $\eta$ and $\beta$ are the relaxation times of the order parameter and the vector potential, respectively. We have solved the TDGL equations upon using the link variables method. [13, 14, 15] Since we consider invariance of the system along the $z$ direction, our approach could only be applied to a square mesoscopic superconducting cylinder. However, it might also be used for a very thin superconducting film of thickness $d \leq \xi(0)(d \leq 1$ in dimensionless units), as long as we replace $\kappa^{2}$ in Eq. 1 by $\kappa_{\text {eff }}=\kappa^{2} / d$. [16] The geometry we have considered is depicted at the top left corner of Fig. 1\} the lateral size of the sample is $d_{S}$, and of the antidot $d_{A D}$.

For the study presented in this Letter, the relaxation times were kept fixed at $\eta=\beta=1$. The GL parameter was also maintained at $\kappa_{\text {eff }}=25$, corresponding, for instance, to a very thin film of $\mathrm{Nb}$ with thickness $d \approx$ $7.3 \mathrm{~nm}$ (assuming $\xi(0)=40 \mathrm{~nm}, \kappa=2.125, \kappa_{\text {eff }}=$ $\left.\kappa^{2} /(d / \xi(0)) \approx 25\right)$.

For $T=0.53$, Fig. 1 shows a short hysteresis loop which was made for a mesoscopic superconducting square (hereafter referred to as sample $S_{1}$ ) of dimensions $d_{S}=$ 12 , with a small antidot at the center, with size $d_{A D}=2$. Points marked with letters, $(a), \ldots,(f)$, indicate the values of $H$ where a vortex (antivortex) either enters or exits the sample. The magnetic field is increased from zero until a value somewhat above that at which the first two vortices penetrate in $(a)$. It is then reversed until the opposite value is achieved, and then reversed once more. At point $(d)$, two antivortices penetrate the superconductor. Points $(b)$ and $(e)$ correspond to the exit of a vortex and an antivortex, respectively, immediately after which one flux quantum still remains in the antidot. The point we will be mainly focusing on is $(c)$ [or, equivalently, $(f)$ ]. This point corresponds to the entrance of an antivortex, which will encounter a vortex trapped in the antidot. The V-AV pair will annihilate either inside or outside the antidot.

We have made a systematic study of the V-AV annihilation process, varying the experimentally accessible

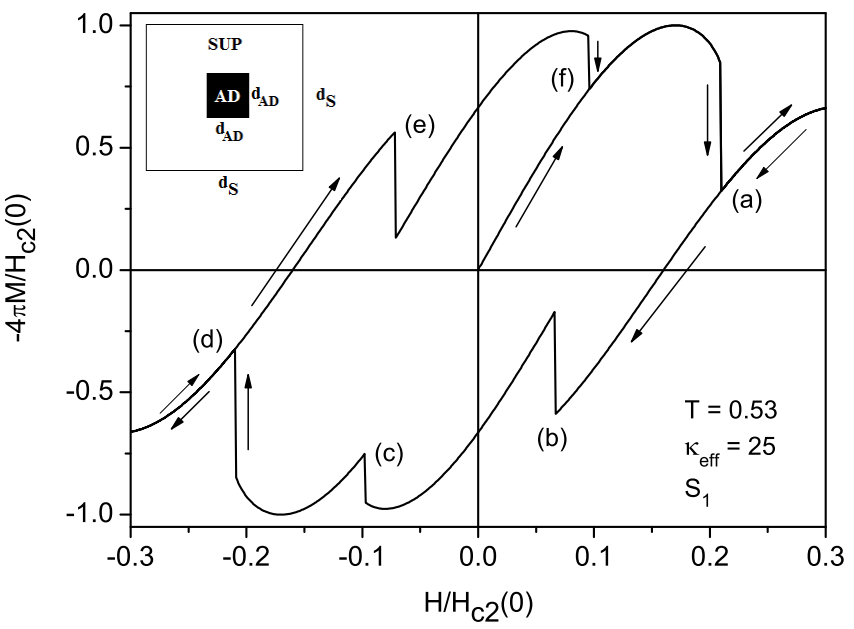

FIG. 1: The magnetization curve as a function of the external applied magnetic field normalized to its maximum value. The meaning of the points marked in the figure are explained in the text. The arrows indicate the direction of the hysteresis loop. The inset illustrates the geometry of the problem.

relevant parameters: $T, d_{S}$, and $d_{A D}$. The other parameters were kept fixed as specified above.

We turn now into a brief discussion of our results. Firstly, we found that for $d_{S}$ and $d_{A D}$ fixed, the VAV annihilation occurs at a limited temperature interval. Above the upper limit of such interval, the vortex trapped at the antidot leaves the sample before the entrance of the antivortex, and no collision can possibly occur. For the sample $S_{1}$ this upper threshold is somewhat above $T=0.53$. As a matter of fact, since the penetration length increases as the temperature approaches $T_{c}$, the supercurrent density associated with the pinned vortex spreads to distances large enough to reach the external border of the sample. This enhances an attractive interaction between the surface and the trapped vortex, facilitating its escape.

At a given temperature, there is a minimum width of the superconducting frame to allow for a V-AV collision outside the antidot. For example, for $T=0.23$ and $d_{A D}=2$ the minimum size of the frame [17] is 6 (these dimensions define sample $S_{2}: d_{S}=8$ and $d_{A D}=2$ ). Our reasoning is as follows: the antivortex nucleated at the external border is kept pinned by the surface barrier at the external border while, in turn, the vortex remains pinned at the antidot. Both entities attract each other and the interaction is larger for smaller superconducting frames. It is then easier to pull the vortex out of the antidot for shorter distances. On the other hand, if the superconductor width is increased, the antivortex starts its excursion toward the center before the V-AV attraction becomes appreciable. Being thus less anchored by the surface, the antivortex travels all the way to the antidot and the annihilation process completes there. In support to this argument, Fig. 2 depicts the values of 
the $x$ component of the supercurrent density at the surfaces $y=12$ and $y=8$ for both samples $S_{1}$ and $S_{2}$, respectively. These values of $J_{s x}$ were calculated in the absence of applied field, $H=0$, and thus represent the supercurrent due only to the vortex inside the antidot. The V-AV attraction (Lorentz force) will be proportional to $J_{s x}$. Notice that, for both samples, the absolute value of $J_{s x}$ is maximum at the center of the square edge. One can clearly see that, in the middle of the sample edge, $x=d_{S} / 2$, the intensity of the Lorentz force is systematically larger for the sample $S_{2}$ than for $S_{1}$.

Fig. 3 shows the dynamics of the V-AV collision for the sample $S_{1}$ at $T=0.53$. Immediately after entering the sample, only the antivortex is in the superconducting sea. As it moves forward to the antidot, at some instant the trapped vortex will come out and both will collide and annihilate.

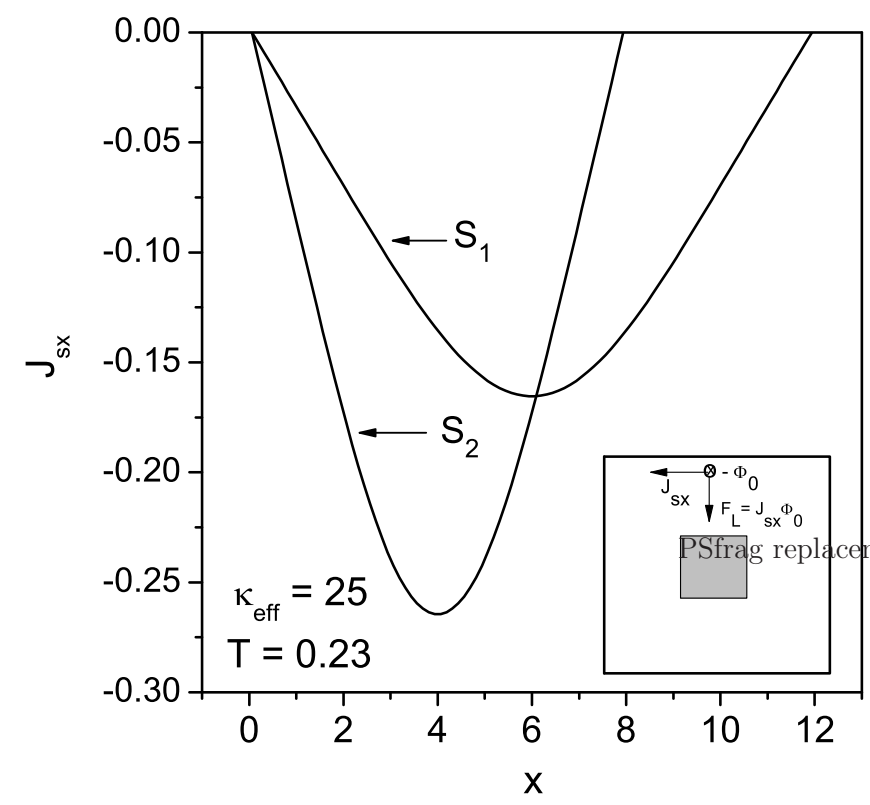

FIG. 2: The supercurrent density along the top surface of the superconductor. The parameters used are specified in the figure. The inset shows the Lorentz force on the antivortex due to the vortex in the antidot.

Another interesting characteristic we have found was that as the antivortex penetrates, its interaction with the vortex is so strong that both appear entirely deformed. Usually, vortices look like a round flux filament surrounded by whirling supercurrents. However, when the vortex and the antivortex approach one another while still partially pinned at the corresponding surfaces, they become alongated, as if squashed, and form a narrow channel between the external surface and the antidot. This can be seen in Fig. 4, which represents the topology of the order parameter $\psi$ and the supercurrent distribution at the very moment when the collision takes place for the sample $S_{1}$ at $T=0.53$. It is very suggestive that, during the collision time, supercurrents nearly van-
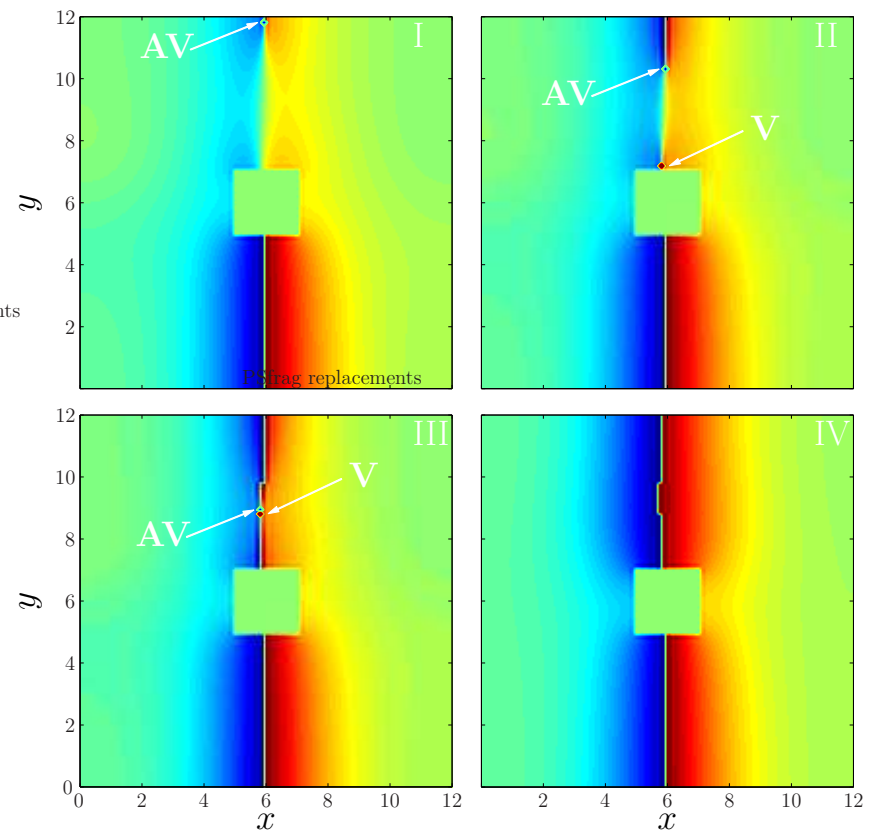

FIG. 3: (Color online) The pictures show the topological shape of the phase of the order parameter; the phase goes from $-\pi$ (blue) to $\pi$ (red). The last picture (IV) corresponds to the stationary state of point $(c)$ of the hysteresis loop of Fig. 1.

ish accross the line of the elongated pair $\mathrm{V}-\mathrm{AV}$, just like in a phase-slip event [18], which is the dual process of Josephson tunneling.
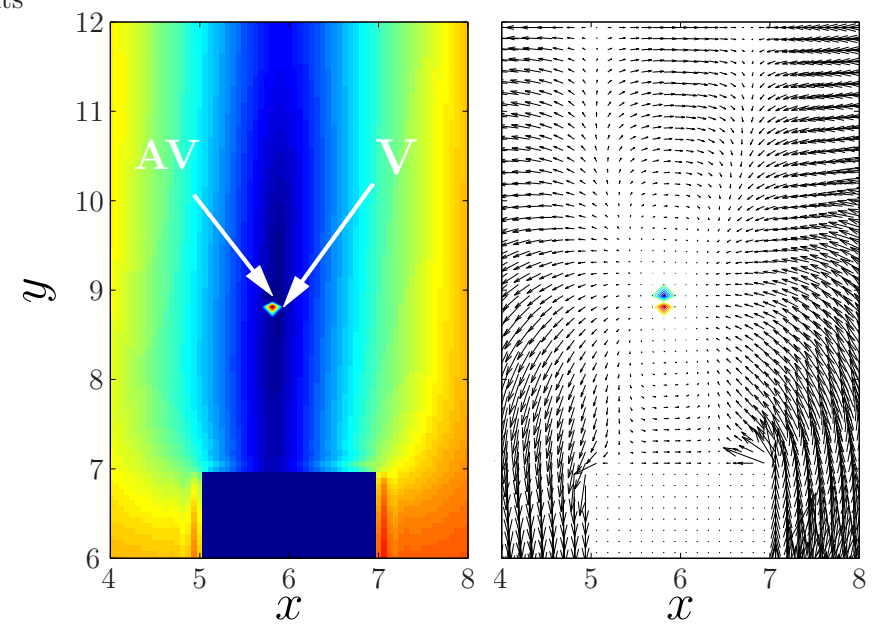

FIG. 4: (Color online) The absolute vale of the order parameter (left) and the supercurrent distribution (right) for the sample $S_{1}$; very dark color means order parameter very depreciated. The size of the arrows (right) are not real; they were enlarged for better viewing.

We also have estimated the instantaneous velocity of the vortex on its way from nucleation at the surface through its capture by the antidot (point (a) of Fig. 1). In addition, the velocities of both the antivortex entering 


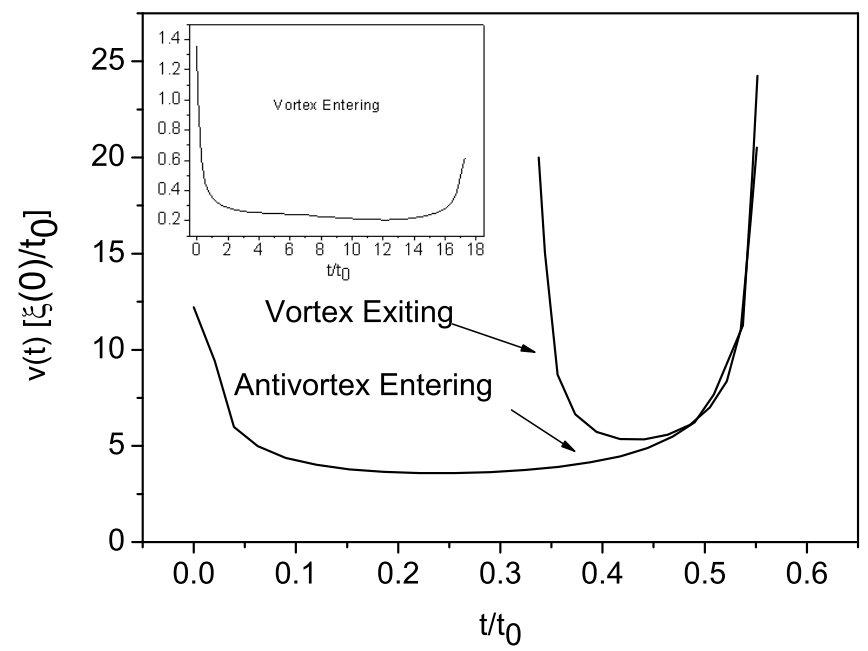

FIG. 5: The modulus of the velocity of the vortex and the antivortex for the sample $S_{1}$ at $T=0.53$ as a function of time (point (c) of Fig. 1); the inset corresponds to point $(a)$.

the sample and that of the vortex exiting the antidot, on their route to annihilation (point (c) of Fig. (1), were also determined. The results are shown in Fig. 5, It is clearly seen that the velocities achieved during the collision process are much larger than those of a vortex penetrating the sample (at least an order of magnitude, as shown in the inset).

In Reference [19] (see also 20, 21]) it was estimated that a phase-slip line can achieve a velocity which is two orders of magnitude higher than an Abrikosov vortex which, in turn, is one order of magnitude faster than Josephson vortices. As Abrikosov vortices are quoted 19] to travel at $10^{3} \mathrm{~m} / \mathrm{s}$, this means that typical velocities for a phase-slip line are of the order of $10^{5} \mathrm{~m} / \mathrm{s}$. Although there is a conceptual difference between the narrow channel of Fig. 4 (a composition of a vortex and an antivortex) and a phase-slip line, the similarity in format is reinforced by the fact that their velocities are approximately the same. For instance, for the sample $S_{1}$, the window during which the antivortex remains visible is $\Delta t=0.5517 t_{0}$ and the distance it travels is $\Delta y=2.875 \xi(0)$; inserting $T_{c}=3.72 \mathrm{~K}$ and $\xi(0)=230 \mathrm{~nm}$ (the relevant parameters for Sn, which were used by Sivakov et al. [19]), the average velocity is $v_{\mathrm{AV}}=1.5 \times 10^{5} \mathrm{~m} / \mathrm{s}$. On the other hand, for the vortex exiting the antidot we obtain $\Delta t=0.2135 t_{0}$ and $\Delta y=1.625 \xi(0)$; which gives $v_{\mathrm{Vs}}=2.2 \times 10^{5} \mathrm{~m} / \mathrm{s}$. Thus, in terms of format and velocity, the annihilation process could be envisaged as a phase slip. It is worth noticing that the large velocities anticipated for the V-AV pair along the collision process, are similar to those developed during the early stage of a vortex avalanche, as observed by the authors of Ref. [6], who reported velocities as large as $1.8 \times 10^{5} \mathrm{~m} / \mathrm{s}$ for avalanches in YBCO films.

For the vortex first entering the antidot we found an average velocity slightly larger then that for a normal Abrikosov vortex: $\Delta t=17.2792 t_{0}, \Delta y=4.125 \xi(0)$, which yields $v_{\mathrm{Ve}}=6.8 \times 10^{3} \mathrm{~m} / \mathrm{s}$. A value somewhat larger - although in the same order of magnitude - might be attributed to the additional attraction exerted by the antidot.

In summary, we have studied the dynamics of the a V-AV annihilation. The process was activated magnetically through a short hysteresis magnetization loop. Our results indicate that the $\mathrm{V}-\mathrm{AV}$ collision can take place either inside the antidot or at the superconducting sea. In addition, we have shown that, during the annihilation process, vortices and antivortices may be stretched by a competition between their mutual attraction and the forces that act to keep them anchored at the surfaces. To complete the picture, we have also reported velocities of the entities while travelling through the superconducting frame of the sample.

The authors thank the Brazilian Agencies FAPESP, FACEPE, and $\mathrm{CNPq}$ for financial support.

[1] C. A. Duran, P. L. Gammel, R. E. Miller, and D. J. Bishop, Phys. Rev. B 52, 75 (1995).

[2] P. Leiderer, J. Boneberg, P. Brull, V. Bujok, and S. Herminghaus, Phys. Rev. Lett. 71, 2646 (1993).

[3] T. H. Johansen, M. Baziljevich, D. V. Shantsev, P. E. Goa, Y. M. Galperin, H. N. Kang, H. J. Kim, E. M. Choi, M. S. Kim, et al., Superconductor Science \& Technology 14, 726 (2001).

[4] F. Colauto, E. M. Choi, J. Y. Lee, S. I. Lee, V. V. Yurchenko, T. H. Johansen, and W. A. Ortiz, Superconductor Science \& Technology 20, L48 (2007).

[5] F. Colauto, E. J. Patino, M. G. Blamire, and W. O. Ortiz, Superconductor Science \& Technology 21, 045018 (2008).

[6] B. Biehler, B.U. Runge, P. Leiderer, and R. G. Mints, Phys. Rev. B 72, 024532 (2005); B.U. Runge, U. Bolz, J. Eisenmenger, and P. Leiderer, Physica C 341-348, 2029 (2000).

[7] G. R. Berdiyorov, M. V. Milošević, and F. M. Peeters, Phys. Rev. Lett. 96, 207001 (2006).

[8] R. Geurts, M. V. Milošević, and F. M. Peeters, Phys. Rev. Lett. 97, 137002 (2006).

[9] V. R. Misko, V. M. Fomin, J. T. Devreese, and V. V. Moshchalkov, Phys. Rev. Lett. 90, 147003 (2003).

[10] A. S. Melnikov, I. M. Nefedov, D. A. Ryzhov, I. A. Shereshevskii, V. M. Vinokur, and P. P. Vysheslavtsev, Phys. Rev. B 65, 140503(R) (2002).

[11] L. F. Chibotaru, A. Ceulemans, V. Bruyndoncx, and V. V. Moshchalkov, Nature (London) 408, 833 (2000).

[12] N. R. Weethamer in Superconductivity, edited by R. D. Parks (New York, 1969).

[13] W. D. Groop. H. G. Kaper, G. K. Leaf, D. M. Levine, M. Palumbo, and V. M. Vinokur, J. Comput. Phys. 123, 254 (1996).

[14] G. C. Buscaglia, C. Bolech, and A. López in Connectivity and Superconductivity, edited by J. Berger e J. Rubinstein (Springer, Heidelberg, 2000). 
[15] E. Sardella, A. L. Malvezzi, P. N. Lisboa-Filho, and W. A. Ortiz, Phys. Rev. B 74, 014512 (2006).

[16] D. J. Priour and H. A. Fertig, Phys. Rev. B 67, 054504 (2003).

[17] While changing sample and antidot sizes, we have always adopted unitary steps.

[18] J. E. Mooij, and Yu. V. Nazarov, Nature Physics 2, 169 (2006).
[19] A. G. Sivakov, A. M. Glukhov, A. N. Omelyanchouk, Y. Koval, P. Müller, and A. V. Ustinov, Phys. Rev. Lett. 91, 267001 (2003).

[20] A. Andronov, I. Gordion, V. Kurin, I. Nefedov, and I. Shereshevsky, Physica C 213C, 193 (1993).

[21] S. G. Doettinger, S. Kittelberger, R. P. Huebener, and C. C. Tsuei, Phys. Rev. B 56, 14157 (1997). 